Laser Chem., 1999, Vol. 19, pp. 145-148

Reprints available directly from the publisher Photocopying permitted by license only
(C) 1999 OPA (Overseas Publishers Association) N.V. Published by license under the Harwood Academic Publishers imprint, part of The Gordon and Breach Publishing Group.

\title{
TIME-RESOLVED TERAHERTZ SPECTROSCOPY OF CONDENSED PHASE REACTIONS
}

\author{
RICHARD MCELROY and KLAAS WYNNE* \\ Femtosecond Research Centre, Dept. of Physics and Applied Physics, \\ University of Strathclyde, Glasgow, G4 ONG, UK
}

(Received 7 April 1997)

Ultrafast time-resolved visible-pump, far-IR $(\mathrm{THz})$ probe spectroscopy has been developed in our lab and has been applied to study carrier dynamics in photoexcited GaAs and dipole solvation dynamics in betaine and $p$-nitroaniline. This type of spectroscopy enables us to study for the first time the nonequilibrium interaction between excited electronic states and low frequency vibrational modes.

Keywords: Ultrafast; spectroscopy; condensed phase; dynamics; terahertz; semiconductors

Ultrafast optical Kerr effect and photon echo experiments [1] have shown that there is a correlation between the rates of simple chemical reactions and low frequency $\left(10-100 \mathrm{~cm}^{-1}\right)$ librational modes present in polar solvents and proteins. However, no true microscopic identification of the relevant modes has been made, as these experiments are only sensitive to the equilibrium solvent spectrum. Time-resolved $\mathrm{THz}$ pump-probe spectroscopy may be more suitable, as it is in principle sensitive to the non-equilibrium dynamics of the solvent modes. We have developed a system for ultrafast visible/nearUV pump, THz-probe spectroscopy. Pump-probe experiments were performed on the carrier dynamics of photoexcited SI GaAs and the dynamic dipole solvation of two different dyes in polar solution.

\footnotetext{
* Corresponding author. Tel.: + 44(141) 548-3381; Fax: +44(141) 552-2891; e-mail: k.wynne@strath.ac.uk
} 
Femtosecond pulses are generated by a Coherent Mira/RegA laser system resulting in $120 \mathrm{fs}$ pulses at $800 \mathrm{~nm}$ with $4 \mu \mathrm{J} /$ pulse at a repetition rate of $250 \mathrm{kHz}$. Nearly single cycle THz pulses with a $200 \mathrm{fs}$ cycle length, a peak frequency of $50 \mathrm{~cm}^{-1}$ and a usable bandwith from 1 to $85 \mathrm{~cm}^{-1}$, are generated and electro-optically detected in $\mathrm{ZnTe}$ $[2,3]$. The experimental setup has two optical delay lines, one for the pump-probe delay and one for the gate delay, i.e., the time delay within the $\mathrm{THz}$ probe pulse. At each pump-probe delay, the gate delay line can be scanned and the data Fourier transformed to obtain a transient spectrum. Pump-probe experiments were performed on SI GaAs with the pump centered at 800 or $400 \mathrm{~nm}$ (see Fig. 1). The creation of carriers by the pump pulse gives rise to a transient absorption over the entire accessible frequency range that can be described by a modified Drude-Lorentz model based on a generalized Langevin equation (see Fig. 1). Pumping SI GaAs at $400 \mathrm{~nm}$ forces the

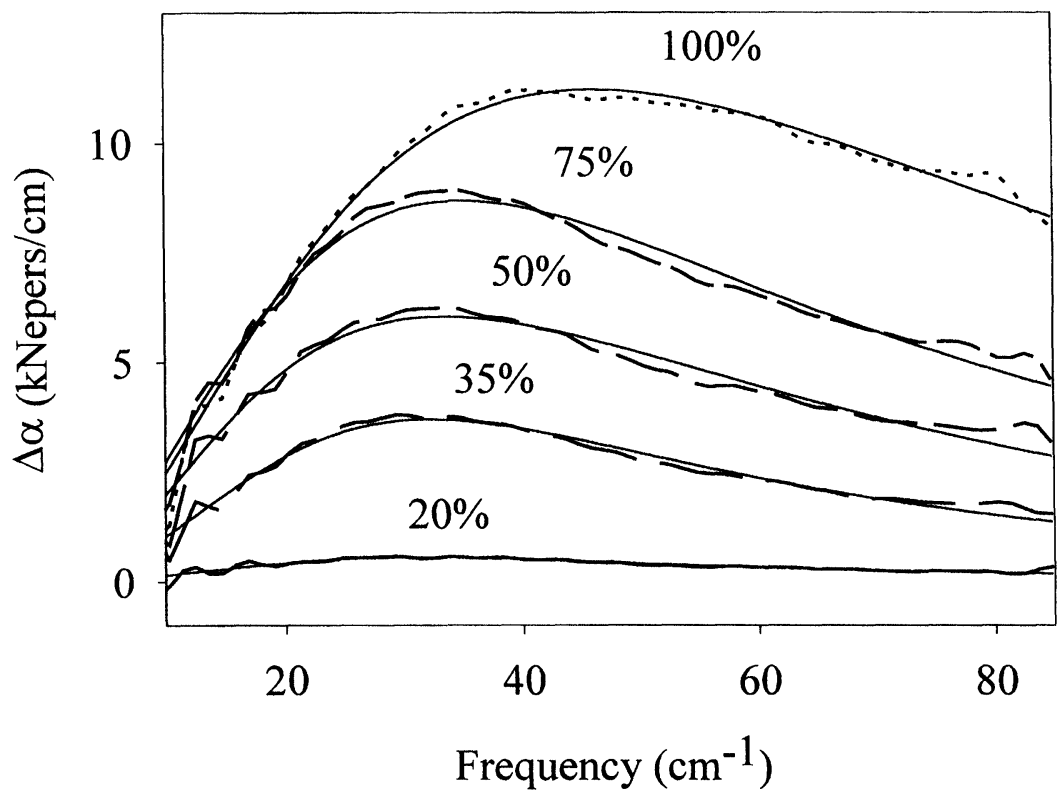

FIGURE 1 Transient spectra due to free carrier absorption photoinduced by an $800 \mathrm{~nm}$ pump pulse in SI GaAs. The spectra were taken at a pump-probe delay of $20 \mathrm{ps}$ and at pump powers varying from full to $20 \%$. All traces were fitted to a modified Drude-Lorentz absorption model. 
carriers into the $\mathrm{L}$ and $\mathrm{X}$ valleys; Relaxation, due to the emission of phonons, back into the main $\Gamma$ valley is observed to take place in $1.35 \pm 0.06 \mathrm{ps}$.

As model systems for condensed phase chemical reactions, the electron-transfer reaction in betaine [4] and the charge-shift reaction in $p$-nitroaniline (PNA) were used. Betaine undergoes an electrontransfer reaction in circa 1 ps from $S_{1}$ back to $S_{0}$ in which the dipole moment changes by 9D [5]. PNA has a dipole moment that increases by 7.3 $\mathrm{D}$ upon photoexcitation [6] and an excited state lifetime of circa $25 \mathrm{ps}$ in $\mathrm{m}$-dichlorobenzene. Figure 2 shows the change in the $\mathrm{m}$ dichlorobenzene far-IR absorption after excitation of PNA at $400 \mathrm{~nm}$ [7]. In both betaine and PNA an oscillatory component is seen with a frequency of $4.0 \pm 0.7 \mathrm{~cm}^{-1}$ and a decay time of $2.0 \pm 0.8 \mathrm{ps}$. The data taken in PNA show a bleach of the ground state absorption that grows with $6.9 \pm 3.3 \mathrm{ps}$ and that does not decay within the observed time window. Static absorption spectra of betaine and PNA in various

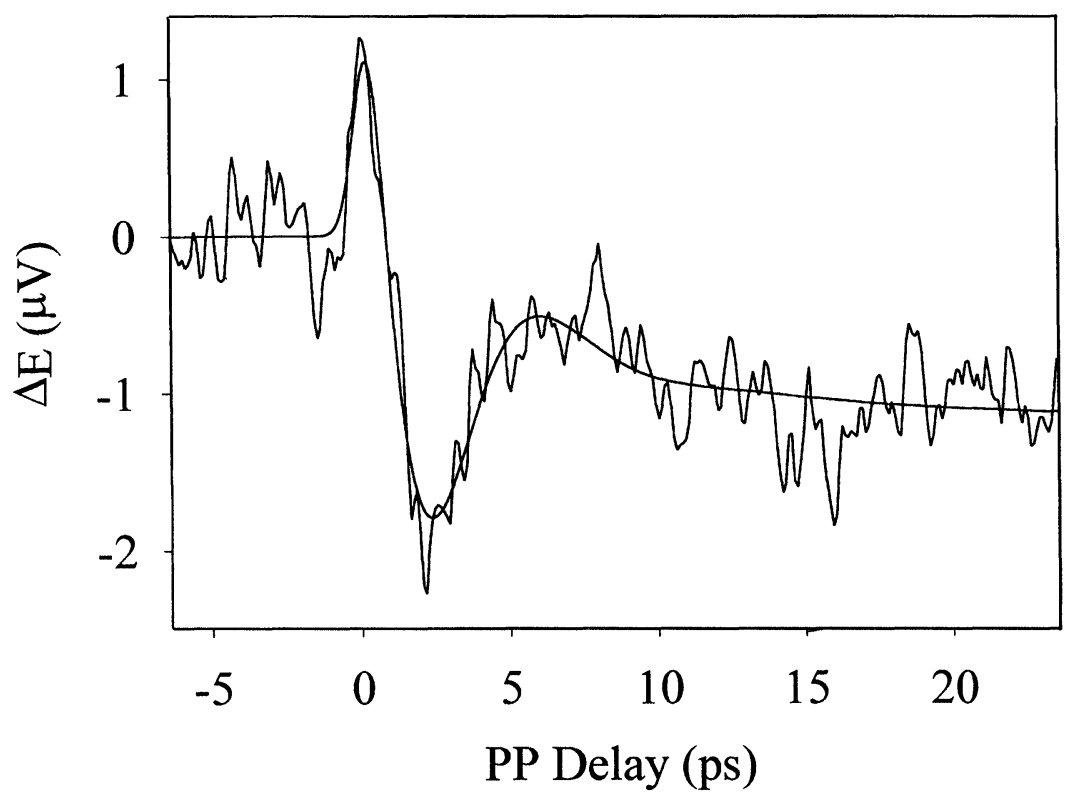

FIGURE 2 Transient pump-probe signal taken in $p$-nitroaniline in $m$-dichlorobenzene. The pump is at $400 \mathrm{~nm}$ (resonant with $\mathrm{S}_{1} \leftarrow \mathrm{S}_{0}$ transition of PNA) and the probe is a THz pulse monitored at its peak for various pump-probe delays. 
polar solvents show that there is a small blue shift of the far-IR absorption band due to the presence of the dyes. Hence, the persistent bleach observed in the pump-probe signal in PNA may be due to a semi-permanent rearrangement of the solvent molecules around the solute in its polar excited state. Within the signal-to-noise, no such persistent signal is observed in betaine consistent with its short $4.3 \mathrm{ps}$ excited state lifetime in $m$-dichlorobenzene [4]. We surmise that the oscillatory signal is an impulsive response of the solvent molecules in the first solvation shell to the sudden dipole moment change upon photoexcitation of the solute.

\section{References}

[1] Vöhringer, P., Arnett, D. C., Westervelt, R. A., Feldstein, M. J. and Scherer, N. F. (1995). J. Chem. Phys., 102, 4027.

[2] Zhang, X.-C. and Jin, Y. (1994). In: Perspectives in Optoelectronics, Edited by Jha (World Scientific), 81.

[3] Wu, Q., Litz, M. and Zhang, X.-C. (1996). Appl. Phys. Lett., 68, 2924.

[4] Åkeson, E., Johnson, A. E., Levinger, N. E., Walker, G. C., DuBruil, T. P. and Barbara, P. F. (1992). J. Chem. Phys., 96, 7859.

[5] Liptay, W. (1969). Angew. Chem. Int. Edit., 8, 177.

[6] Sinha, H. K. and Yates, K. (1991). J. Am. Chem. Soc., 113, 6062.

[7] McElroy, R. and Wynne, K. (1997). Phys. Rev. Lett., 79, 3078. 